\title{
Homeostasis and the concept of 'interstitial fluids hierarchy': Relevance of cerebrospinal fluid sodium concentrations and brain temperature control (Review)
}

\author{
LUIGI F. AGNATI ${ }^{1,2}$, MANUELA MARCOLI ${ }^{3}$, GIUSEPPINA LEO ${ }^{1}$, GUIDO MAURA $^{3}$ and DIEGO GUIDOLIN ${ }^{4}$ \\ ${ }^{1}$ Department of Biomedical Sciences, University of Modena and Reggio Emilia, 41121 Modena, Italy; \\ ${ }^{2}$ Department of Neuroscience, Karolinska Institutet, 17177 Stockholm, Sweden; ${ }^{3}$ Department of Pharmacy, \\ Unit of Pharmacology and Toxicology, and Center of Excellence for Biomedical Research, University of Genova, \\ 16126 Genova; ${ }^{4}$ Department of Molecular Medicine, University of Padova, 35121 Padova, Italy
}

Received August 11, 2016; Accepted December 12, 2016

DOI: $10.3892 / \mathrm{ijmm} .2017 .2874$

\begin{abstract}
In this review, the aspects and further developments of the concept of homeostasis are discussed also in the perspective of their possible impact in the clinical practice, particularly as far as psychic homeostasis is concerned. A brief historical survey and comments on the concept of homeostasis and allostasis are presented to introduce our proposal that is based on the classical assumption of the interstitial fluid (ISF) as the internal medium for multicellular organisms. However, the new concept of a hierarchic role of ISF of the various organs is introduced. Additionally, it is suggested that particularly for some chemico-physical parameters, oscillatory rhythms within their proper set-ranges should be considered a fundamental component of homeostasis. Against this background, we propose that the brain ISF has the highest hierarchic role in human beings, providing the optimal environment, not simply for brain cell survival, but also for brain complex functions and the oscillatory rhythms of some parameters, such as cerebrospinal fluid sodium and brain ISF pressure waves, which may play a crucial role in brain physio-pathological states. Thus, according to this proposal, the brain ISF represents the real internal medium since the maintenance of its dynamic intra-set-range homeostasis is the main factor for a free and independent life of higher vertebrates. Furthermore, the evolutionary links between brain and kidney and their synergistic role in $\mathrm{H}_{2} \mathrm{O} / \mathrm{Na}$
\end{abstract}

Correspondence to: Professor Luigi F. Agnati, Department of Biomedical Sciences, University of Modena and Reggio Emilia, via Giuseppe Campi 287, 41125 Modena, Italy

E-mail: luigiagnati@tin.it

Dr Diego Guidolin, Department of Molecular Medicine, University of Padova, via Gabelli 65, 35121 Padova, Italy

E-mail: diego.guidolin@unipd.it

Key words: brain, $\mathrm{H}_{2} \mathrm{O} / \mathrm{Na}$ balance, integrative actions, interstitial fluid, kidney, psychic homeostasis, temperature balance and brain temperature control are discussed. Finally, it is surmised that these two interrelated parameters have deep effects on the Central Nervous System (CNS) higher integrative actions such those linked to psychic homeostasis.

\section{Contents}

1. A general premise and the aims of the present article

2. Historical aspects

3. The fluid compartments of the brain

4. Evolutionary aspects of brain/kidney interactions

5 Heuristic hypothesis

6. Discussion

\section{A general premise and the aims of the present article}

Homeostasis is a fundamental concept in physiology; hence, it is a subject of the greatest interest for medicine (1-3). In the present review, some aspects of the concept and possible further developments that could have an impact also in the clinical practice are analysed.

Our proposal is based on the classical assumption of the interstitial fluid (ISF) as the internal medium for multicellular organisms. Against this background, a central aspect is discussed; namely the concept of the hierarchic role of the ISF of the various organs in triggering homeostatic responses, especially of those involving overriding controls. In particular, we propose that the brain ISF has the highest hierarchic role in human beings, providing the optimal environment for brain cell survival and complex functions, hence allowing the Central Nervous System (CNS) to carry out its complex whole-body integrative actions, i.e., to operate as the uppermost controller that allows a free and independent life of higher vertebrates. Against this background, the crucial role of the kidneys for internal medium homeostasis will be revised according to the proposal that Homer Smith put forward more than fifty years ago in an extraordinary book (4). As a matter of fact, Homer Smith pointed out, on the basis of evolutionary observations, 
that the functional interconnections between the brain and kidneys in the internal medium homeostasis are a basic requisite for a free and independent life of higher vertebrates. Hence, it can be surmised that CNS operates as the uppermost controller of the internal medium homeostasis not exclusively but especially thanks to its synergistic interactions with the kidney. The clinical evidence supports such a view as discussed below and reported in recent studies $(5,6)$. In particular, in this review, the brain/kidney synergistic roles in $\mathrm{H}_{2} \mathrm{O} / \mathrm{Na}$ balance and brain temperature control is discussed. Actually, data are presented supporting the view that these two parameters are interrelated and have deep effects on the CNS higher integrative actions. Furthermore, the concept of allostasis, hence of a set-range of optimal values for internal medium parameters is assumed (7). This view is further developed by considering the possible functional meaning of the rhythmic oscillations of the chemico-physical parameters inside their set-range that, according to our proposal, should be considered as a basic component of the homeostatic control.

Thus, the present proposal suggests that ISF represents the real internal medium, since the maintenance of its homeostasis and in several instances the appropriate rhythms of some chemico-physical parameters within their appropriate set-range is the main factor for a free and independent life of higher vertebrates.

In particular, the main focus of the present review is the suggestion that $\mathrm{H}_{2} \mathrm{O} / \mathrm{Na}$ balance and brain temperature play a crucial role in certain brain integrative actions. A special emphasis will be given to their rhythmic oscillations within their set-range that can have important modulatory actions on psychic homeostasis. As a matter of fact, psychic homeostasis (see section below entitled 'Body and psychic homeostasis' for a definition) refers to complex behavioural and bodily responses optimizing, inter alia, the interactions of a human subject with his socio-cultural environment (i.e., the so-called supra-systems). It should be underlined that psychic homeostasis can sometimes override bodily homeostasis regulatory mechanisms, i.e., the controls aimed to optimize human being interactions with his external physical environment $(7,8)$.

Body and psychic homeostasis. The need to characterize the complexity of the human being has led to a distinction between two elements: the soma and psyche (9). Thus, it has been proposed that two highly complementary homeostatic control processes are in operation in human beings: i) the somatic physiological homeostasis, which via the integrative actions of the different apparatuses is capable of maintaining important chemico-physical parameters of the internal milieu within optimal set-ranges, allowing a free and independent life to the organism; and ii) the psychic homeostasis, which is aimed to an equilibrated mental condition via a proper resetting action of the different instances of the inner world and social environment that the subject has to harmonize. In agreement with such a view, Freud proposed the principle of 'psychic homeostasis' as a basic concept supporting his meta-psychology in his famous book 'The Interpretation of Dreams' (10).

To indicate the psychic homeostasis in its proper set-range (corresponding to a psychic state of subjective well-being) the term eudaimonia (also mentioned as eudaemonia), that consists of the words 'eu' ('good') and 'daimōn' ('spirit'), has been proposed. Thus, it could be described as a condition of dynamic balance between different appeals within the psychic sphere of the subject also in front of challenges of the social and physical environment.

These aspects were further expanded (11) into the concept of 'predictive psychic homeostasis' that designates the capability to create (via the integrated actions of various brain-body mechanisms) a psycho-physical state of readiness that anticipates potential threats. Thus, predictive psychic homeostasis by avoiding possible future allostatic psycho-physical overloads is of a basic relevance for both eudaimonia and internal milieu homeostasis $(8,11)$. The survival value of predictive psychic homeostasis is emphasised by the evidence that it can sometimes override bodily homeostasis regulatory mechanisms $(7,8)$.

It should be noted that both body homeostasis and psychic homeostasis are often jointly conditioned by the pattern of endocrine signaling $(11,12)$. In particular, alterations of anterior pituitary hormones leading to somatic disturbances have been demonstrated in stress and mood disorders, including major depression (13). Thus, hypothalamic-pituitary-adrenocortical hormones have deep effects on both body homeostasis and psychic homeostasis and it is interesting to point out that both are modulated by circadian secretion rhythms $(14,15)$.

In other words, human CNS high integrative actions link together human basic physiology with the subject's supra-systems that play a basic role in psychic homeostasis. Thus, for the human being psychic homeostasis and bodily homeostasis are two inseparable aspects of his health. Such a view reminds aphorisms of ancient medicine and philosophy that have proposed the soul serenity (Eudaimonia, i.e., the good spirit; see also the Discussion) as the primary aim of subject's well-being. Obviously, Eudaimonia is deeply dependent on gratifying interactions between the individual human being and his socio-cultural environment $(16,17)$.

\section{Historical aspects}

The French physiologist Claude Bernard (1813-1878) introduced the concept of the internal environment of an organism (18), upon which Walter Cannon (1871-1945) proposed the concept of homeostasis. A crucial aspect is obviously the definition of internal medium and the characterization of its distinctive features in physiological terms (19).

Initially Bernard postulated that blood was the milieu interieur, or internal environment, exchanging substances and operating as an optimal environment for cells metabolism of higher animals. Thus, he proposed the concept of the fixity or constancy of the internal environment as essential to the vital processes by stating: 'The fixity of the milieu supposes a perfection of the organism such that the external variations are at each instant compensated for and equilibrated. The stability of the internal environment is the condition for the free and independent life' (20).

Later on the interstitial fluid, as the environment with which the cells of a multicellular organism exchange, was proposed and the concept of 'homeostasis' was introduced by Cannon. The new term was defined as follows (21): 'The constant conditions which are maintained in the body might be termed equilibria. That word, however, has come to have fairly exact meaning as applied to relatively simple physico-chemical 
states, in closed systems, where known forces are balanced. The coordinated physiological processes which maintain most of the steady states in the organism are so complex and so peculiar to living beings - involving, as they may, the brain and nerves, the heart, lungs, kidneys and spleen, all working cooperatively - that I have suggested a special designation for these states, homeostasis. The word does not imply something set and immobile, a stagnation. It means a condition - a condition which may vary, but which is relatively constant. Homeostasis, at least in the narrow sense, is about preserving the status quo, regulation to achieve viability through resistance to change.' Furthermore, Cannon adapted the idea of homeostasis to the organism as a whole, hence referring to both physiological and psychological aspects of control mechanisms. As mentioned above, this broadening of the homeostasis concept to the psychic aspects is of peculiar relevance for the human being and it will be considered in the present review.

Let us discuss with some detail the concept of allostasis that was introduced in 1988 by Sterling (7), as a further development of some aspects of the concept of homeostasis (22). In fact, allostasis points out that the living systems are endowed with the adaptability property, hence the control mechanisms operate for a continuous adjusting to change, not simply by restoring, but rather by altering equilibrium settings in order to face in an optimal fashion the new environmental inputs. Thus, not a set-point for the controlled parameter but rather a set-range is maintained (Fig. 1). In other words, allostasis describes the processes through which organisms actively maintain a dynamic steady state of conditions for basic parameters necessary not simply for their survival but rather for optimal interactions with the environment.

It should be underlined that allostatic mechanisms can also adapt the organisms to probable future loads (Bayesian approach) and this is obviously especially true for organisms that are endowed with a complex CNS. Thus, the concept of allostasis was introduced to underline both physiological and psychological change and adaptation to diverse present and surmised future circumstances. Obviously, the behavioural and physiological anticipatory responses to probable future events emphasize the crucial role of a complex CNS, particularly for responses allowing the so-called predictive allostasis (1,7-8,16,23-26).

We should also mention another far-sighted proposal of Cannon, since he underlined that the internal environment with which the cells exchange is not simply a watery environment but rather a 'fluid matrix' whose chemical and also physical characteristics, such as temperature and pressure, are of a basic importance.

It should be noted that the fluid matrix [hence the aqueous extracellular matrix (ECM)] forming the internal medium is the product of the organism itself and it can play a basic role in the intercellular communication processes (27-30). The evidence that the internal medium and in particular the ECM is produced by the organism according to its functional needs implies a broader view of homeostasis, since the self-construction of the internal medium is in some way related to concepts introduced in the 1970s by Varela et al (31) and discussed by others by stating (31-33): 'A living system is a system capable of self-reproduction and self-maintenance through a regenerative network of processes which take place within a boundary of its own making and regenerates itself through cognitive or adaptive interactions with the medium' (33).

Thus, a living system can be characterized by two notions (33-35): i) the first one is 'autopoiesis', a term indicating the ability of the system to transform external matter/energy into an internal process of self-maintenance and production of its own components. Autopoiesis, therefore, is concerned with internal structure and describes a system organized as a network of interactions capable of cyclically reproducing the components to allow the system's self-perpetuation; and ii) the second one can be called 'cognition' to indicate that the interaction with the environment is implemented on the basis of the internal logic of the living system. The environment, in other words, does not prescribe or determine changes to the organism, it is the structure of the living system and its previous history of perturbations that determine what reactions a new perturbation will induce. Cognition, therefore, concerns the relationship of the system with the environment. It describes a system able to sense the external world in order to produce a response to perturbations either by self-correction of its disturbed organization (homeostasis), or by reorganizing itself into a different self-perpetuating network of interactions (adaptability). There are, of course, various levels of cognition (corresponding to different levels of life's complexity) from very simple mechanisms of cell sentience (36) up-to complex functions performed by nervous systems.

This view captures the essence of cellular life by recognizing that life is a cyclic process that produces the components that in turn self-organize in the process itself, and all within a boundary of its own making. Such a self-constructed machine continuously operates to maintain critical chemico-physical parameters within a suitable set-range in spite of perturbing influences.

As pointed out by Wiener in his classical book (37), the mechanisms allowing the processes of maintenance of the values of critical parameters within suitable set-ranges that allow survival of the living beings notwithstanding the external environment changes can be described according to cybernetic models.

Mechanisms allowing homeostasis: contributions from cybernetics. During the middle of the past century, Norbert Wiener (1894-1964) pointed out that the concept of homeostasis was common to animals and to automatic systems (37). Thus, he defined cybernetics as the entire field of the theory of communication and control in the living beings and in the automatic systems. During the 1940's and 1950 's, cybernetics had a crucial influence on the birth of various modern sciences: control theory, computer science, information theory, automata theory, artificial intelligence and artificial neural networks, cognitive science, computer modelling and simulation science, dynamical systems, and artificial life. However, after these disciplines had become fully independent, cyberneticists felt the need to further develop their field to distinguish an approach emphasizing autonomy, self-organization, cognition from more mechanistic control strategies followed in control engineering and computer science. In the early 1970's, this effort became known as 'second-order cybernetics' (38) and provided a thoughtful revision of concepts of particular relevance for biology and physiology. 

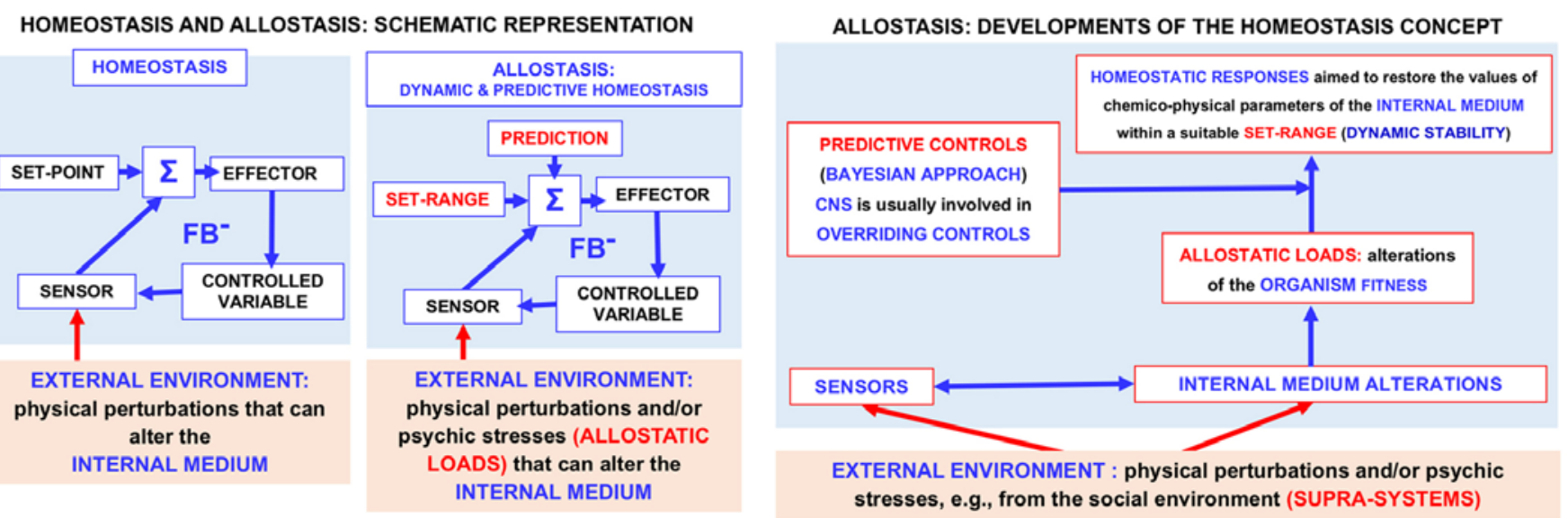

Figure 1. Schematic representation of the concepts of homeostasis and allostasis. The panel on the right clarifies the important developments that the allostasis concept has introduced moving from the concept of homeostasis. For further details, see text. FB ${ }^{-}$, negative feedback. $\Sigma$ indicates any set of processes integrating and processing the incoming input to provide the output.

In particular, the negative feedback $\left(\mathrm{FB}^{-}\right)$is a type of control central to both homeostasis and cybernetics, since it can explain how a living being or an automatic system can operate for maintaining its steady state in spite of disrupting influences from the external environment (Fig. 1). The FB' control can be described as follows: let us suppose that a stress from the external environment acts upon a system of an organism and such a system is under a FB $^{-}$control. A sensor detects the stress signal and converts it into signals that are meaningful for the internal control mechanisms of the organism. A controller compares the signals to the desired set-points, and issues error signals if there are discrepancies. Thus, each error signal refers to the difference between the optimal value of a variable (set-point) and the value returned by the homeostatic mechanism(s) during the persisting perturbation. The error is interpreted by an activator, which triggers the appropriate response of effectors to restore the value of the variable close to its set-point or, according a more realistic view, within a suitable set-range (Fig. 1).

As pointed out above, in a living being, several regulatory mechanisms are continuously activated to produce the homeostatic responses; hence, in analyzing the allostatic controls the following aspects should at least be considered: i) redundancy and inter-dependence, since several negative feedbacks are directly or indirectly involved in the control of the same functional parameter or of functional parameters strictly interdependent (e.g., blood volume and arterial blood pressure); ii) push-pull controls (e.g., glucagon/insulin) allowing a precise control of the parameter; and iii) Russian Doll organization of controls of increasing miniaturization (39-41). Thus, it is possible to recognize $\mathrm{FB}^{-}$control at the systems (set of apparatuses) level, at the level of organs, at the level of tissues, at the level of the cells, at the level of the molecular networks.

The crucial role for overriding controls is played by the CNS via its interactions with the peripheral apparatuses especially for parameters, such as arterial blood pressure, involved in facing challenging conditions (e.g., in fight or flight conditions) (42-48).

Taken together, it can be stated that homeostasis implies the regulated activation of several dynamic and potentially predictive multilevel processes organized according to a Russian Doll pattern $(32,42,43,49,50)$. Furthermore, as proposed by 'brain-body medicine',the CNS plays a crucial role in homeostasis by interacting with practically all the peripheral organs $(42,51-53)$.

In this context, the brain/kidney interactions for the $\mathrm{H}_{2} \mathrm{O} / \mathrm{Na}$ and brain temperature control that are interrelated parameters of basic importance for CNS integrative actions, will be discussed. As discussed below, such an assumption can also be surmised by deductions based on the evolutionary background.

\section{The fluid compartments of the brain}

As stated in the general premise, it is proposed that the brain ISF plays the highest hierarchic role among the ISF of the different organs of the human body in triggering homeostatic responses of fundamental importance. Such a strong assumption does not deny the relevance of the holistic view of the human body as proposed by 'brain-body medicine' (42), but it is aimed at suggesting a frame to organize the data indicating that the ISF dyshomeostasis reduces to a great extent the CNS integrative actions that allow a free and independent life. As mentioned above, to support our assumption, we focus our analysis on two parameters, namely the $\mathrm{H}_{2} \mathrm{O} / \mathrm{Na}$ and brain temperature control and the $\mathrm{CNS} /$ kidney synergistic interactions.

As an introduction to this subject, a preliminary analysis is proposed on the complex fluid and cellular movements among the various brain fluid compartments, since they are involved not only in the metabolic support to neurons and glial cells and in the clearance of waste molecules from the brain, but also in the brain interactions with the systemic apparatuses. In an interesting study, Brinker et al published a very useful diagram showing the complex fluid and cellular exchanges between the brain fluid compartments (54) and we will refer to it for our proposal.

Actually, according to our view, it is possible to organize the brain fluid compartments distinguishing a 'major cerebrospinal fluid (CSF) circulation' (black arrows in Fig. 2) from an 'inter-compartments fluid circulation' (blue arrows in Fig. 2) from a 'CSF intra-ventricular local circulation' 


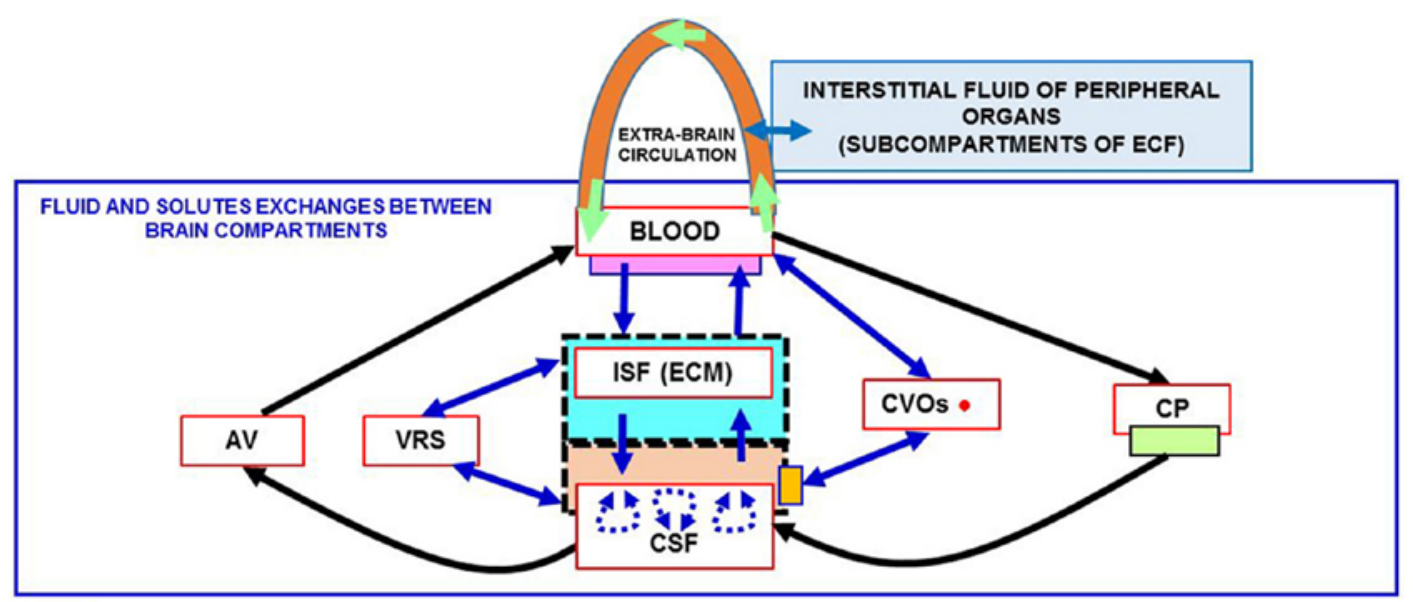

\section{BLOOD-BRAIN BARRIER}

EPENDYMA (highly permeable gap junctions in the pia and ependyma that allow free exchange of solutes I. paracellularly between brain ISF and ventricular CSF)

CELL-INTERSTITIUM RELATIONSHIPS (tissue microarchitecture, e.g., tortuosity and viscosity of the brain ECM)

TANYCYTES (tanycyte-like cells display well-organized tight junctions around their cell bodies. Thus, this ependymal layer acts as a diffusion barrier)

CP BARRIER

$\leftrightarrow$ INTER-COMPARTMENTS CIRCULATION (exchanges of water, solutes and in some instances also of cells)

- $\left[\mathrm{Na}^{+}\right]$sensors \& TRP channels for the osmosensors

1) CSF MAJOR CIRCULATION (CP secretes CSF that flows through the ventricles into the subarachnoid space to arachnoid granulations. The exit of CSF is mainly to the blood. CSF operates as 'nourishing liquor' and also as long-distance channel for VT signals migration)

: CSF INTRA-VENTRICULAR LOCAL CIRCULATION (bidirectional ebbs and flows that create loca

.... mixing especially close to the ventricular walls. It operates also as local communication channel for VT signals migration)

Figure 2. Schematic representation of the main fluid compartments of the brain. It should be noted that both CSF and ISF are separated by the cerebral circulation (blood) by selective barriers while can communicate in a rather easy way with each other. Furthermore, the scheme indicates that within the cranial theca at least three different modes of fluid movements can be recognized: exchange of fluids and in some instances also of cells between compartments; major CSF circulation that is CP secretes CSF that flows through the ventricles into the subarachnoid space to arachnoid granulations to have the main exit mainly to the blood; intra-ventricular local circulation of CSF that is characterized by bidirectional ebbs and flows that create local mixing especially close to the ventricular walls. AV, arachnoid villi; CP, choroid plexus; CSF, cerebrospinal fluid; CVOs, circumventricular organs; ECF, extracellular fluid; ECM, extracellular matrix; ISF, brain interstitial fluid; TRP, transient receptor potential; VRS, Virchow-Robin space; VT, volume transmission.

characterized by gentle ebbs (broken arrows in Fig. 2). The schematic representation suggests a special hub status for ISF in view of its multiple connections with the other fluid compartments. Furthermore, a privileged status for ISF is pointed out by the limiting barriers that regulate its cellular and fluid exchanges with the other compartments. Actually, ISF is protected by a highly selective barrier from the cerebral blood circulation and by a permeable barrier formed by gap junctions in the pia and ependymal layer and by the brain tissue microarchitecture (e.g., tortuosity and viscosity of the brain ECM) from CSF. Furthermore, it should also be pointed out that ISF and Virchow-Robin space (VRS) are separated only by the glial barrier through which also cells can move to ISF.

As indicated in Fig. 2, CSF operates as an interface between the major and minor circles. In addition, CSF exchanges signals with ISF not only directly but also indirectly via its reciprocal interactions with the VRS. Thus, CSF secretion, composition and circulation have a central importance for ISF homeostasis. Let us analyze some aspects of CSF secretion, composition and circulation.
As far as the secretion of CSF is concerned, the choroid plexus (CP) has been proposed as the main locus for the secretion and clearance of CSF. Thus, at CP the blood-CSF barrier secretes and purifies CSF, generates intracranial pressure and maintains the CSF composition. Therefore, the CP secretory epithelium plays a basic role for brain homeostasis and integrative actions via multiple transport and secretory functions that support CSF homeostasis and its fluid dynamics (55).

As far as CSF composition is concerned, it is important to compare at least some of its main chemico-physical parameters with those of plasma and extracellular fluid (ECF). Two highly selective barriers separate CSF and ISF from the blood, while they are separated from each other by easily permeable boundaries. Thus, if ISF has the highest hierarchic order among the brain ISF, CSF should be also considered in view of its continuous renewal and circulation as a crucial fluid compartment for brain integrative functions and as a good mirror of brain ISF. On the other hand, plasma is the weighted mean of the different ISF of the various organs. Each of these will contribute to plasma composition according 
Table I. Chemico-physical parameters characterizing ECF, plasma and CSF (87-89).

\begin{tabular}{lccc}
\hline Parameter & ECF & Plasma & CSF \\
\hline $\mathrm{Na}^{+}(\mathrm{mEq} / \mathrm{l})$ & $136-145$ & 150 & 147 \\
$\mathrm{~K}^{+}(\mathrm{mEq} / \mathrm{l})$ & $3.5-5$ & 4.6 & $\mathbf{2 . 9}(\mathbf{0 . 6 2})$ \\
$\mathrm{Ca}^{2+}(\mathrm{mEq} / \mathrm{l})$ & 3.4 & 4.7 & $\mathbf{2 . 3}(\mathbf{0 . 4 9})$ \\
$\mathrm{Mg}^{2+}(\mathrm{mEq} / \mathrm{l})$ & $1.50-2.5$ & 1.6 & $\mathbf{2 . 2}(\mathbf{1 . 3 9})$ \\
$\mathrm{Cl}^{-}(\mathrm{mEq} / \mathrm{l})$ & $\mathbf{1 1 0 - 1 1 8}$ & 105.0 & $\mathbf{1 2 0}(\mathbf{1 . 1 4})$ \\
$\mathrm{HCO}_{3}{ }^{-}(\mathrm{mEq} / \mathrm{l})$ & $22-28$ & 24.8 & 25.1 \\
$\mathrm{pH}$ & $7.35-7.45$ & $7.38-7.42 \mathrm{~A}-\mathrm{WB}$ & 7.4 \\
$\mathrm{P}_{\mathrm{O} 2}(\mathrm{mmHg})$ & $\mathbf{3 5}$ & $75-100 \mathrm{~A}-\mathrm{WB}$ & $\mathbf{4 2}$ \\
$\mathrm{P}_{\mathrm{CO} 2}(\mathrm{mmHg})$ & 39.5 & $35-45 \mathrm{~A}-\mathrm{WB}$ & $\mathbf{5 0 . 2}$ \\
$\mathrm{Glucose}(\mathrm{mg} / \mathrm{ml})$ & $70-110$ & $70-110$ & $\mathbf{6 4}$ \\
Osmolality $\left(\mathrm{mOsm} \mathrm{kg}{ }^{-1} \mathrm{H}_{2} \mathrm{O}\right)$ & $280-296$ & $280-296$ & 289 \\
Temperature $\left({ }^{\circ} \mathrm{C}\right)$ & $36.6-37.3$ & 37.0 & $\mathbf{3 7 . 7}$ \\
\hline
\end{tabular}

A-WB: Arterial whole blood; ECF (extracellular fluid ) and CSF (cerebrospinal fluid) values different from plasma are indicated in bold font.

to its volume, composition and access to plasma. Thus, brain ISF also in view of the blood-brain barrier (BBB) will not greatly contribute to plasma composition notwithstanding its high hierarchic order.

These aspects are illustrated in Fig. 2 and in Table I where some of the main chemico-physical parameters of plasma, ECF and CSF are presented.

As far as CSF circulation i.e., the 'third circulation' according to Cushing (56) is concerned, it has been shown that adult CP secretes CSF that flows through the ventricles into the subarachnoid space, then over the top of the brain, with some CSF flowing down to the lumbar sac. The exit of CSF is mainly to the blood at the arachnoid granulations but also to the lymph system through the nasal cribriform plate or via the spinal nerve roots (55).

However, CSF flow is not unidirectional since it has been shown that not only is a pulsatile flow but also characterized by up and down movements throughout the entire brain with local fluid exchanges with ISF, circumventricular organs (CVOs) and VRS.

Furthermore, it should be underlined that the CSF has not only gentle and bidirectional ebbs and flows that create mixing (55), but it has also been shown that the single layer of ciliated ependymocytes in contact with the CSF beat in a periodic manner, generating localized microscale (beating of cilia) effects on near-wall CSF dynamics (Fig. 2). This action may contribute in clearing debris from the ventricle walls, and may enhance mixing, which may be particularly relevant for neuroendocrine communication (57) or even for signal detection (36). Thus, while some short-distance volume transmission (VT)-signalling can rely upon this action of the ependymal cilia close to the walls of lateral ventricles, long-distance VT-signalling could be mainly driven by wall motion and $\mathrm{CP}$ pulsation, hence by CSF dynamics in the centre regions of the ventricles, according to the tide hypothesis (58). Thus, it has been suggested to distinguish a major CSF circulation that is the classical one from the $\mathrm{CP}$ to the arachnoid granulations, from a local CSF micro-circulation that is mainly confined close to the ciliated walls of ventricles. While the former plays a role as nourishing liquor and long-distance pathway for VT signal migration, the latter one is mainly involved in short distance migration of VT signals and CSF flow-mediated neuronal guidance $(57,59,60)$.

Furthermore, the CSF circulation around blood vessels penetrates from the subarachnoid space into the VRS allowing cell exchange between blood and brain. As proposed by Bechter et al (60-62), CSF is also a diffusion pathway for pathogenic agents causing neuropsychiatric diseases (63). Actually, it has been shown that blood-borne inflammatory cells enter the brain via VRS or via CP and clinical evidence supports the relevance of an immunological crosstalk between periphery and intrathecal immunity in neurological and psychiatric diseases $(54,64)$.

The fluid movements at the barriers are driven by osmotic and hydrostatic gradients or by active transporter processes. Aquaporins and other transporters play a fundamental role in the control of fluid exchange at the glial, endothelial, and CP barrier. In particular, the water influx into the VRS and, hence, interstitial flow is regulated by aquaporin- 4 localized in the end feet of astrocytes (65). It is interesting to note that the fluid movements into and out of the VRS depend on respiratory and cardiac pressure pulsations and can be an important component of the tide hypothesis for VT signal diffusion in the brain (58).

A special role of tanycytes for brain-body interactions has also been proposed, since tanycyte-like cells are a characteristic feature of all CVOs and they display well-organized tight junctions around their cell bodies that can represent a BBB displaced from the vascular to the ventricular side (66). Thus, tanycytes may play an important role in regulating the exchange between blood, CSF and ISF, and hence in regulating the brain-body interactions, particularly in some organs that are deeply involved in the control of internal medium as the kidney.

The assumption of peculiar brain/kidney interactions finds an indirect support from comparative physiology and evolutionary studies. 


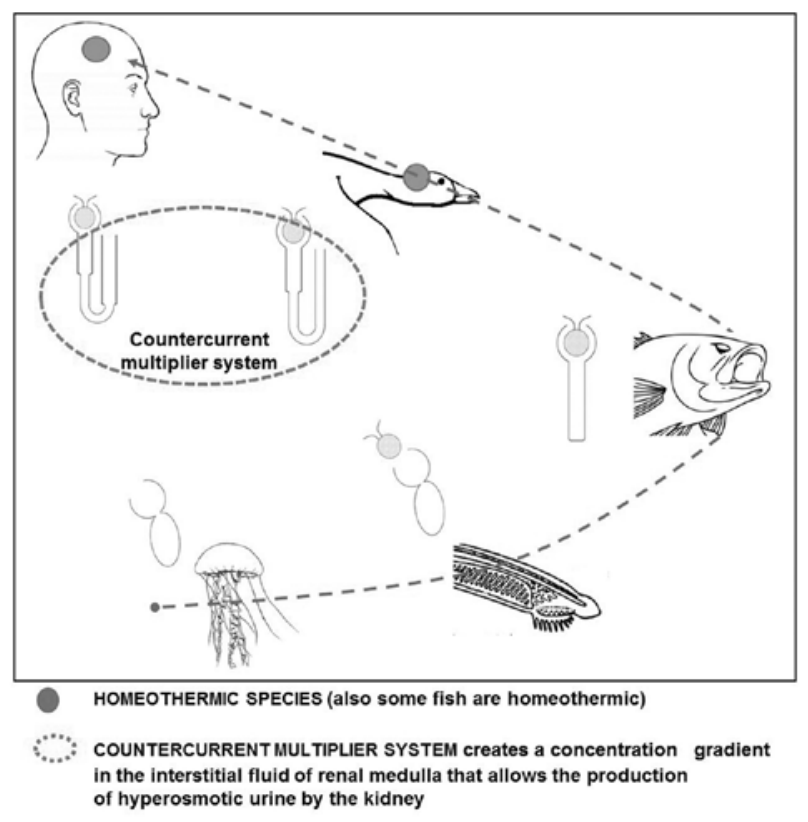

Figure 3 . The present figure is inspired by a schematic illustration that Homer Smith (1953) devised to clarify the evolutionary and functional links between complexity of the nervous system and complexity of the kidneys (83). As indicated in the figure, in the present review, it is noted that the homeostasis of body temperature is to a certain extent associated with the parallel evolution of the brain and kidneys. In fact, in our opinion, a precise control of the volume and composition of the internal medium has allowed the establishment of a complex nervous system capable of complex dynamic predictive homeostasis especially for chemico-physical parameters that are highly relevant for central nervous system integrative actions such as the sodium concentrations and brain temperature.

\section{Evolutionary aspects of brain/kidney interactions}

In the famous and fascinating book cited above, Homer Smith (4) proposed that the following processes do indicate a parallel evolution of the nervous system and kidneys (Fig. 3): i) the increased complexity of the renal apparatus has allowed a progressively more precise control of the volume and composition of the internal medium; ii) the precise control of the volume and composition of the internal medium has allowed the establishment of a progressively more complex nervous system; and iii) the organism endowed with a complex CNS has become capable of multidimensional allostatic controls, i.e., of complex dynamic predictive homeostasis, particularly for chemico-physical parameters that are highly relevant for CNS integrative actions.

Accordingly, some aspects will be discussed as to how a complex CNS operates to allow interconnections between the brain and kidneys for the homeostatic controls of sodium concentrations and brain temperature.

As far as plasma sodium concentrations are concerned it should be noted that this parameter not only has a restrict set range $\left(135 \mathrm{mEq} / 1<\left[\mathrm{Na}^{+}\right]<147 \mathrm{mEq} / \mathrm{l}\right)$, but also that hyponatremia causes important CNS symptoms (e.g., amnesias, headaches, epilepsy) (5).

As far as cerebral temperature is concerned, it should be noted that brain deep structures are at $1-2^{\circ} \mathrm{C}$ above the body core temperature. Thus, blood in the cerebral circle is at a a lower temperature than the deep brain structures hence cere- bral blood flow besides providing nutrients to the brain and allowing the clearance of its metabolic waste products has also the function of absorbing heat especially from the active brain regions (67-70).

The osmotic regulation of water homeostasis and body core temperature are functionally related, since the maintenance of body water homeostasis is critical for preventing hyperthermia, as evaporative cooling is the most efficient means of dissipating excess body heat.

As far as water homeostasis is concerned, its control is mainly achieved by the regulation of water intake and water loss by the kidneys. The former is achieved by sensations of thirst that motivate water intake, whereas the latter is regulated by the antidiuretic action of vasopressin. Vasopressin (also known as antidiuretic hormone (ADH) (5) secretion and thirst are stimulated by increases in the osmolality of the ECF, as well as by decreases in blood pressure and/or blood volume, signals that are precipitated by water depletion associated with the excess evaporative water loss required to prevent hyperthermia. Thus, it can be deduced that thermoregulation and osmotic regulation of water homeostasis are functionally interdependent.

Let us briefly mention two important recent contributions on the mechanisms involved in this integrated homeostatic control $(71,72)$.

It has been demonstrated that thermo-sensitive neurons in the preoptic-anterior hypothalamus are modulated by $\mathrm{ADH}$. Actually, ADH enhances thermo-sensitivity of warm-sensitive neurons and decreases thermo-sensitivity of cold-sensitive neurons. Such ADH modulatory actions promote heat loss and are probably involved in the $\mathrm{ADH}$-induced hypothermia (71).

The basic role of ADH for both osmotic regulation of water homeostasis and cerebral homoeothermic control are schematically summarized in Fig. 4.

Another mechanism involved in the integration of thermal and the osmotic regulation of water homeostasis has also been described, that is based on the transient receptor potential-vanilloid (TRPV) family of ion channels that respond both to osmotic signals and also are stimulated by increases in body temperature (72).

As described in the recent study by Noda et al (6), at least two independent sensing systems in the CVOs are involved in monitoring osmolality and body-fluid volume, namely $\mathrm{Na}(\mathrm{x})$ channels and TRP channels. In particular, $\mathrm{Na}(\mathrm{x})$ channels have been demonstrated to play a role as $\left[\mathrm{Na}^{+}\right]$sensors and TRP channels as osmosensors, but these channels are also temperature-sensitive. Let us briefly describe these two molecular mechanisms involved in the control of $\mathrm{Na} / \mathrm{H}_{2} \mathrm{O}$ and cerebral temperature: i) $\mathrm{Na}(\mathrm{x})$ channels are located in perineuronal processes and in the ependymal cells of the subfornical organ and organum vasculosum lamina terminalis (OVLT) and are activated by hypernatremia. Their activation causes a reduction in $\mathrm{Na}$ assumption; and ii) TRPV channels, such as the TRPV1 and TRPV4 are expressed at the level of the supraoptic nucleus and at the level of the OVLT. These channels are activated by hyper-osmolality but also by increases in the cerebral temperature. Their activation causes $\mathrm{Na} / \mathrm{H}_{2} \mathrm{O}$ reabsorption.

In other words, there are brain regions (particularly CVOs) and central molecular mechanisms (e.g., TRPV) allowing 

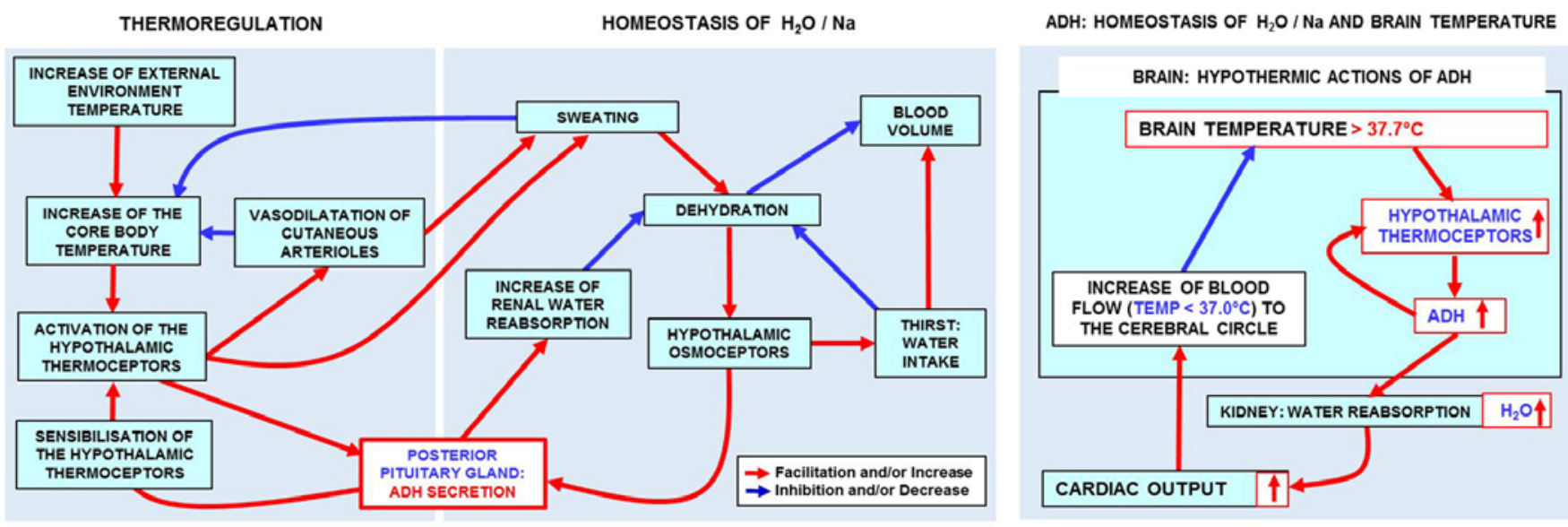

Figure 4. Schematic representation of the mechanisms involved in the synergistic interactions of brain and kidney in the control of brain temperature and of the volume and composition of the internal medium. For further details, see text. ADH, vasopressin.

brain-kidney interactions in the control of ECF osmolality and cerebral temperature (72).

Thus, apart from the mechanism that is dependent on $\mathrm{ADH}$ modulatory actions on hypothalamic thermo-receptors and via such an action integrate thermal and osmotic regulation, the mechanism that is dependent on TPRV channels whose activation is directly modulated by fluid osmolality and cerebral temperature should also be considered.

The triggering of these mechanisms also depends on multiple neural and humoral signals from the periphery. The latter ones can reach the brain via the cerebral circulation and from the blood can reach the brain integrative centres, particulatly those located in the CVOs, since they are outside the BBB (54-66).

\section{Heuristic hypothesis}

In the frame of the basic problem already faced by Claude Bernard, that is of the definition of internal medium and the characterization of its distinctive features in physiological terms, we propose that there is a hierarchic order among the ISF and we maintain that brain ISF is the real 'internal medium' of higher vertebrates since it is the most important ISF for triggering the complex responses allowing a free and independent life and this is particularly true for human beings. As mentioned above, this assumption does not deny the holistic view of the human organism but it gives a further aspect to the "brain-body medicine' that has recently been investigated also for a surprising new aspect, namely the synergistic role between brain and gut microbiota (73). Thus, while the general relevance of the brain/body interactions are a main subject of investigations the main highly focused subject of our paper is how brain circuits and kidney functions can modulate brain ISF composition.

Thus, according to our proposal, brain ISF represents the 'core fluid compartment' that provides an optimal environment for development, survival and, together with CSF, it allows VT intercellular communications among the brain complex cellular networks $(58,74)$. Thus, brain ISF is likely a crucial functional component of the multifaceted integrative CNS actions that are involved in most of the dynamic predictive homeostatic mechanisms and overriding controls. The crucial role of brain ISF is also indicated by the evidence that does exchange signals with the other brain fluid compartments but only in a highly regulated way (Fig. 2). Our view is that brain ISF has the highest hierarchic role in triggering dynamic homeostatic responses involved in the balance of volume and sodium concentrations of the ECF as well as the cerebral temperature control. As discussed above, it is suggested that these two functionally interrelated parameters play a crucial role for the high level integrative actions of CNS as those involved in the control of the psychic state. In agreement with such a view many clinical studies demonstrate correlations between the water/sodium balance, homoeothermic balance and psychic conditions of the subject. Actually, it has been reported that: i) psychic stresses increase the plasma and CSF levels not only of cortisol but also of $\mathrm{ADH}(75)$; ii) $\mathrm{ADH}$ plasma levels are increased in depressed patients (76); iii) epidemiological studies have demonstrated a correlation between depression and habitats with a high salinity (77); iv) schizophrenic patients have alterations in the control of body temperature (78); and v) RPV1 channels beside responding to the osmotic signals are also activated by cerebral temperature $(6,72)$ and at the level of the cerebral cortex are altered in depression (79).

It has been introduced the term of psycho-physic allostasis to indicate the responses aimed not only to the maintenance of the internal medium homeostasis but also to the broader condition of well-being of the subject hence to his Eudaimonia (16,17, see Section 'Body and psychic homeostasis'). This condition of well-being especially depends on the interactions between the systems of the organism and the supra-systems. Thus, Eudaimonia does depend from the interactions between the human being and external physical environment but even more from the interactions of the subject with his socio-cultural environment.

\section{Discussion}

According to our proposal, the brain ISF and its 'mirror fluid' the CSF have the highest hierarchic role since they allow optimal conditions for CNS integrative actions. In particular, body-fluid and core temperature controls are two chemico-physical parameters that are essential for a free and 


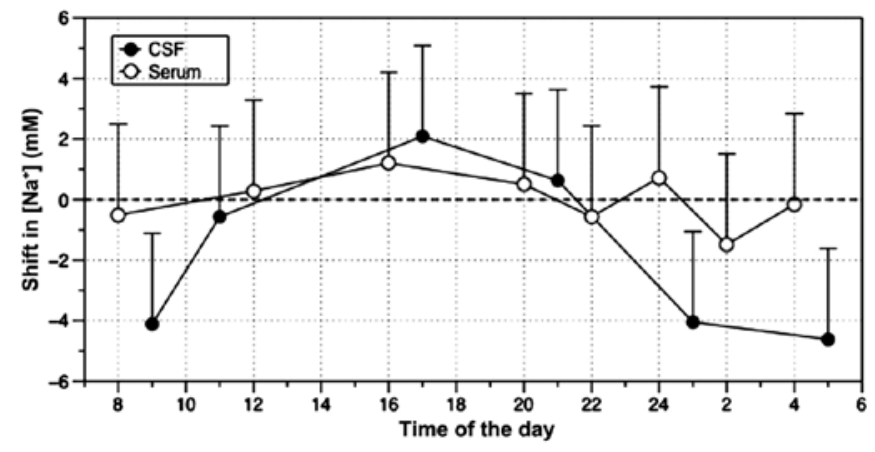

Figure 5. The daily mean rhythm of CSF and serum $\left(\mathrm{Na}^{+}\right)$, plotted as a shift from the mean. CSF data were obtained by Harrington et al (80) from 6 subjects, data in serum by Trotti et al (81) refer to 24 healthy patients. In both studies a statistically significant sodium circadian rhythm was reported However, while plasma levels of $\left(\mathrm{Na}^{+}\right)$exhibited a quite low range of variation during the day (74), the magnitude of change of $\left(\mathrm{Na}^{+}\right)$in CSF was greater (mean shift of about $7 \mathrm{mM}$ ) and sufficient to have physiological effect on neuronal excitability (73). CSF, cerebrospinal fluid.

independent life of human beings that is possible only if their CNS operates in optimal conditions.

Furthermore, we have proposed that in several instances an appropriate oscillatory rhythm (often but not exclusively a circadian rhythm) of a chemico-physical parameter within its appropriate set-range is an essential component of allostasis.

Thus, in several instances the load error is the result of the discrepancy between the internal medium value and not simply the set-range values but the appropriate oscillatory rhythm that the chemico-physical parameter under scrutiny should have.

A consequence of such an assumption is that dyshomeostasis can be actually present even if the chemico-physical parameter under scrutiny is within its appropriate set-range but with inappropriate rhythmic oscillations. Thus, a careful analysis of chemico-physical parameters dynamic patterns should be considered and not only at plasmatic level but also at CSF level. As a matter of fact, it is interesting observing that the concentration rhythms of sodium are differentially regulated (Fig. 5) in the CSF (80) in comparisons with the sodium concentrations rhythms in the systemic circulation (81). This aspect has clinical implications not only since it has been shown that a correlation exists between the rising levels of CSF sodium levels and the timing of migraine onset (80) but also since as recently stated water and sodium homeostasis is inextricably coupled to the CNS integrative actions (82). In this regard, neuroscience describes an inverse relationship between the intensity of the neuropil function and the amount of water it contains with different shrinkage the ECM according to the neuronal activity $(30,82,83)$.

As discussed above, these variations in the ECM perviousness have profound effects on VT signals diffusion $(29,30,74)$ hence also regulate the exchange of signals ISF/blood, ISF/CSF, ISF/VRS. Thus, exchanges between blood-born and brain-born VT signals depend also at some extent on the ECM hydration and composition $(30,82,83)$. This could be one important feature characterizing brain-kidney interactions since as mentioned above integrative centres for the feedback mechanisms involved in $\mathrm{Na} /$ water and cerebral temperature homeostasis are localized at CVOs level that is outside the BBB but separated by barriers from ISF.
Against this background and on the bases of recently published data $(6,72)$ it has been proposed that $\left(\mathrm{Na}^{+}\right)$, osmolality and cerebral temperature are continuously monitored in the brain in particular at CVOs level and are strictly regulated thanks to brain-kidney interactions to maintain the optimal set-range for brain ISF that, in turn, is a necessary prerequisite for the brain integrative actions. Furthermore, on the basis of the clinical data above mentioned it has been proposed that $\left(\mathrm{Na}^{+}\right)$, osmolality and cerebral temperature are interconnected parameters playing a fundamental role in the psychic allostasis. Thus, it is surmised that clinical evaluations of these parameters are of paramount importance for the psychic homeostasis of the subject and could be modulated by optimizing his interactions with the socio-cultural environment in which he lives as well as by psycho-therapy.

In other words, it could be proposed the potential relevance of monitoring internal medium $\left(\mathrm{Na}^{+}\right)$and cerebral temperature during psycho-therapy treatments like the ones administrated to patients suffering of post-traumatic stress disorders $(16,84-86)$. Thus, it could be relevant to evaluate the physical (internal medium parameters) and psychological (Eudaimonia) therapeutic effects produced by addressing the patient's inner speech in such a way of favoring his predictive psychic allostasis to peaceful scenarios. This could lead to a better dealing with the stressing actions of supra-systems and the traumatic memory traces that he has to face $(23,85,86)$.

\section{Acknowledgements}

Professor Agnati is grateful to Professor La Manna for his invitation as a speaker at the Congress of the Accademia Nazionale di Medicina in Bologna (8 October, 2015) where part of this review was presented. This review is dedicated to Carlo Franzini (1942-2015), Professor of the Human Physiology University of Bologna for his important contributions on sleep and blood circulation. He also wrote poems rich of a thoughtful view of human life (see, Carlo Franzini Il Codice di Smirne Book Editore 2002).

\section{References}

1. Schulkin J: Rethinking homeostasis: allostatic regulation in physiology and pathophysiology. MIT Press, Cambridge, 2003.

2. McEwen BS and Wingfield JC: The concept of allostasis in biology and biomedicine. Horm Behav 43: 2-15, 2003.

3. Berntson GG and Cacioppo JT: Integrative physiology: homeostasis, allostasis and the orchestration of systemic physiology. in handbook of psychophysiology. Cambridge University Press 3rd edition: pp433-452, 2007.

4. Smith HW: From Fish to Philosopher. 18th edition, Little, Brown, Boston, 1953.

5. Danziger J and Zeidel ML: Osmotic homeostasis. Clin J Am Soc Nephrol 10: 852-862, 2015.

6. Noda $M$ and Sakuta H: Central regulation of body-fluid homeostasis. Trends Neurosci 36: 661-673, 2013.

7. Sterling $\mathrm{P}$ and Eyer J: Allostasis: A new paradigm to explain arousal pathology: handbook of life stress, cognition, and health. Fisher S and Reason JT (eds). Wiley, Chicester, pp629-649, 1988.

8. Schulkin J: Social allostasis: Anticipatory regulation of the internal milieu. Front Evol Neurosci 2: 111, 2011.

9. Vanzago L: Breve storia dell'anima. Il Mulino, Bologna, 2009 (in Italian).

10. Prata MR: Normal and pathologic in Freud. Physis 9: 37-81, 1999.

11. Borrow AP, Stranahan AM, Suchecki D and Yunes R: Neuroendocrine regulation of anxiety: Beyond the hypothalamic-pituitary-adrenal axis. J Neuroendocrinol 28: 2016. 
12. de Kloet ER, Otte C, Kumsta R, Kok L, Hillegers MH, Hasselmann H, Kliegel D and Joëls M: Stress and depression: A crucial role of the mineralcorticoid receptor. J Neuroendocrinol 28 2016. http://dx.doi.org/10.1111/jne.12379.

13. Bao AM, Meynen G and Swaab DF: The stress system in depression and neurodegeneration: Focus on the human hypothalamus. Brain Res Brain Res Rev 57: 531-553, 2008.

14. Barandas R, Landgraf D, McCarthy MJ and Welsh DK: Circadian clocks as modulators of metabolic comorbidity in psychiatric disorders. Curr Psychiatry Rep 17: 98, 2015.

15. Landgraf D, McCarthy MJ and Welsh DK: Circadian clock and stress interactions in the molecular biology of psychiatric disorders. Curr Psychiatry Rep 16: 483, 2014.

16. Agnati LF, Barlow PW, Baluška F, Tonin P, Guescini M, Leo G and Fuxe K: A new theoretical approach to the functional meaning of sleep and dreaming in humans based on the maintenance of 'predictive psychic homeostasis'. Commun Integr Biol 4: 640-654, 2011

17. Sterling P: Allostasis: A model of predictive regulation. Physiol Behav 106: 5-15, 2012.

18. Bernard C: Introduction à l'étude de la médecine expérimentale. J.B. Baillière et Fils, Paris, 1865.

19. Agnati LF, Fuxe K, Baluska F and Guidolin D: Implications of the 'Energide' concept for communication and information handling in the central nervous system. J Neural Transm Vienna 116: $1037-1052,2009$

20. Bernard C: Lectures on phenomena of life common to animals and plants. JB Balliere and Son, Paris, 1878.

21. Cannon WB: The Wisdom of the Body. Norton \& Company, New York, 1932

22. Day TA: Defining stress as a prelude to mapping its neurocircuitry: No help from allostasis. Prog Neuropsychopharmacol Biol Psychiatry 29: 1195-1200, 2005.

23. Ganzel BL, Morris PA and Wethington E: Allostasis and the human brain: Integrating models of stress from the social and life sciences. Psychol Rev 117: 134-174, 2010

24. McEwen BS and Stellar E: Stress and the individual. Mechanisms leading to disease. Arch Intern Med 153: 2093-2101, 1993.

25. Koob GF and Le Moal M: Plasticity of reward neurocircuitry and the 'dark side' of drug addiction. Nat Neurosci 8: 1442-1444, 2005.

26. Agnati LF, Barlow P, Ghidoni R, Borroto-Escuela DO, Guidolin D and Fuxe K: Possible genetic and epigenetic links between human inner speech, schizophrenia and altruism. Brain Res 1476: 38-57, 2012

27. Agnati LF, Zoli M, Pich EM, Benfenati F and Fuxe K: Aspects of neural plasticity in the central nervous system-VII. Theoretical aspects of brain communication and computation. Neurochem Int 16: 479-500, 1990

28. Agnati LF, Cortelli P, Pettersson R and Fuxe K: The concept of trophic units in the central nervous system. Prog Neurobiol 46: 561-574, 1995

29. Kamali-Zare $P$ and Nicholson C: Brain extracellular space: Geometry, matrix and physiological importance. Basic Clin Neurosci 4: 282-286, 2013 .

30. Marcoli M, Agnati LF, Benedetti F, Genedani S, Guidolin D, Ferraro L, Maura G and Fuxe K: On the role of the extracellular space on the holistic behavior of the brain. Rev Neurosci 26 : 489-506, 2015.

31. Varela FG, Maturana HR and Uribe R: Autopoiesis: The organization of living systems, its characterization and a model. Curr Mod Biol 5: 187-196, 1974

32. Rudrauf D, Lutz A, Cosmelli D, Lachaux JP and Le Van Quyen M: From autopoiesis to neurophenomenology: Francisco Varela's exploration of the biophysics of being. Biol Res 36: 27-65, 2003.

33. Damiano L and Luisi PL: Towards an autopoietic redefinition of life. Orig Life Evol Biosph 40: 145-149, 2010.

34. Thompson E and Varela FJ: Radical embodiment: Neural dynamics and consciousness. Trends Cogn Sci 5: 418-425, 2001.

35. Luisi PL: The emergence of life. From chemical origins to synthetic biology. Cambridge University Press, Cambridge, 2006. http://dx.doi.org/10.1017/CBO9780511817540.

36. Agnati LF, Marcoli M, Maura G, Fuxe K and Guidolin D: The multi-facet aspects of cell sentience and their relevance for the integrative brain actions: Role of membrane protein energy landscape. Rev Neurosci 27: 347-363, 2016.

37. Wiener N: Cybernetics: or control and communication in the animal and the machine. MIT Press, Cambridge, 1948.

38. Heylighen $F$ and Joslyn C: Cybernetics and second-order cybernetics. In: Encyclopedia of physical science \& technology. Meyers RA (ed). Vol. 4, 3rd edition. Academic Press, New York, 2001.
39. Agnati LF and Fuxe K: New concepts on the structure of the neuronal networks: The miniaturization and hierarchical organization of the central nervous system. (Hypothesis). Biosci Rep 4 93-98, 1984.

40. Agnati LF, Genedani S, Leo G, Rivera A, Guidolin D and Fuxe K One century of progress in neuroscience founded on Golgi and Cajal's outstanding experimental and theoretical contributions. Brain Res Brain Res Rev 55: 167-189, 2007.

41. Agnati LF, Guidolin D, Cortelli P, Genedani S, Cela-Conde C and Fuxe K: Neuronal correlates to consciousness. The 'Hall of Mirrors' metaphor describing consciousness as an epiphenomenon of multiple dynamic mosaics of cortical functional modules. Brain Res 1476: 3-21, 2012

42. Agnati LF, Guidolin D, Guescini M, Battistin L, Stocchi V, De Caro R, Genedani S and Fuxe K: Aspects on the integrative actions of the brain from neural networks to 'brain-body medicine'. J Recept Signal Transduct Res 32: 163-180, 2012.

43. Sherrington CS: The Integrative Action of the Nervous System. Charles Scribner's Sons, New York, 1906.

44. Gibson WC: Sir Charles Sherrington, O.M., P.R.S. (1857-1952). In: Twentieth century neurology. The British contribution. Rose FC (ed). Imperial College Press, London, pp1-7, 2002.

45. Gibson WC: A student recalls Sir Charles Sherrington, O.M. (1857-1952). Brain 130: 2766-2769, 2007.

46. Breathnach CS: Charles Scott Sherrington's Integrative action: A centenary notice. J R Soc Med 97: 34-36, 2004.

47. Burke RE: Sir Charles Sherrington's the integrative action of the nervous system: A centenary appreciation. Brain 130: 887-894, 2007.

48. Levine DN: Sherrington's 'The Integrative action of the nervous system': A centennial appraisal. J Neurol Sci 253: 1-6, 2007.

49. Agnati LF, Baluška F, Barlow PW and Guidolin D: Mosaic self-similarity logic, and biological attraction principles: Three explanatory instruments in biology. Commun Integr Biol 2: $552-563,2009$.

50. Agnati LF, Guidolin D and Fuxe K: The brain as a system of nested but partially overlapping networks. Heuristic relevance of the model for brain physiology and pathology. J Neural Transm Vienna 114: 3-19, 2007.

51. Cameron OG: Visceral brain-body information transfer. Neuroimage 47: 787-794, 2009.

52. Lane RD and Wager TD: Introduction to a special issue of Neuroimage on brain-body medicine. Neuroimage 47: 781-784, 2009.

53. Lane RD and Wager TD: The new field of Brain-Body Medicine: What have we learned and where are we headed? Neuroimage 47: 1135-1140, 2009.

54. Brinker T, Stopa E, Morrison J and Klinge P: A new look at cerebrospinal fluid circulation. Fluids Barriers CNS 11: 10 2014.

55. Spector R, Keep RF, Robert Snodgrass S, Smith QR and Johanson CE: A balanced view of choroid plexus structure and function: Focus on adult humans. Exp Neurol 267: 78-86, 2015.

56. Cushing H: Cameron lectures 1 . The third circulation and its channels. Lancet 2: 851-857, 1925

57. Siyahhan B, Knobloch V, de Zélicourt D, Asgari M, Schmid Daners M, Poulikakos D and Kurtcuoglu V: Flow induced by ependymal cilia dominates near-wall cerebrospinal fluid dynamics in the lateral ventricles. J R Soc Interface 11: 20131189,2014

58. Agnati LF, Genedani S, Lenzi PL, Leo G, Mora F, Ferré S and Fuxe K: Energy gradients for the homeostatic control of brain ECF composition and for VT signal migration: Introduction of the tide hypothesis. J Neural Transm Vienna 112: 45-63, 2005.

59. Sawamoto K, Wichterle H, Gonzalez-Perez O, Cholfin JA, Yamada M, Spassky N, Murcia NS, Garcia-Verdugo JM, Marin O, Rubenstein JLR, et al: New neurons follow the flow of cerebrospinal fluid in the adult brain. Science 311: 629-632, 2006.

60. Bechter K: Virus infection as a cause of inflammation in psychiatric disorders. Mod Trends Pharmacopsychiatry 28: 49-60, 2013

61. Bechter K, Reiber H, Herzog S, Fuchs D, Tumani H and Maxeiner HG: Cerebrospinal fluid analysis in affective and schizophrenic spectrum disorders: Identification of subgroups with immune responses and blood-CSF barrier dysfunction. J Psychiatr Res 44: 321-330, 2010.

62. Bechter K and Benveniste H: Quinckes' pioneering 19th centuries CSF studies may inform 21th centuries research. Neurol Psychiatry Brain Res 21: 79-81, 2015. 
63. Tarasoff-Conway JM, Carare RO, Osorio RS, Glodzik L, Butler T, Fieremans E, Axel L, Rusinek H, Nicholson C, Zlokovic BV, et al: Clearance systems in the brain-implications for Alzheimer disease. Nat Rev Neurol 11: 457-470, 2015.

64. Maxeiner HG, Marion Schneider E, Kurfiss ST, Brettschneider J, Tumani $\mathrm{H}$ and Bechter $\mathrm{K}$ : Cerebrospinal fluid and serum cytokine profiling to detect immune control of infectious and inflammatory neurological and psychiatric diseases. Cytokine 69: 62-67, 2014.

65. Nakada T: Virchow-Robin space and aquaporin-4: New insights on an old friend. Croat Med J 55: 328-336, 2014.

66. Langlet F, Mullier A, Bouret SG, Prevot V and Dehouck B Tanycyte-like cells form a blood-cerebrospinal fluid barrier in the circumventricular organs of the mouse brain. J Comp Neurol 521: 3389-3405, 2013.

67. Yablonskiy DA, Ackerman JJ and Raichle ME: Coupling between changes in human brain temperature and oxidative metabolism during prolonged visual stimulation. Proc Natl Acad Sci USA 97: 7603-7608, 2000.

68. Kiyatkin EA, Brown PL and Wise RA: Brain temperature fluctuation: a reflection of functional neural activation. Eur J Neurosci 16: 164-168, 2002.

69. Kozak LR, Bango M, Szabo M, Rudas G, Vidnyanszky Z and Nagy Z: Using diffusion MRI for measuring the temperature of cerebrospinal fluid within the lateral ventricles. Acta Paediatr 99 237-243, 2010.

70. Wang H, Wang B, Normoyle KP, Jackson K, Spitler K Sharrock MF, Miller CM, Best C, Llano D and Du R: Brain temperature and its fundamental properties: A review for clinical neuroscientists. Front Neurosci 8: 307, 2014.

71. Tang Y, Yang YL, Wang N, Shen ZL, Zhang J and Hu HY: Effects of arginine vasopressin on firing activity and thermosensitivity of rat PO/AH area neurons. Neuroscience 219 10-22, 2012.

72. Sladek CD and Johnson AK: Integration of thermal and osmotic regulation of water homeostasis: The role of TRPV channels. Am J Physiol Regul Integr Comp Physiol 305: R669-R678, 2013.

73. Foster JA, Lyte M, Meyer E and Cryan JF: Gut microbiota and brain function: An evolving field in neuroscience. Int J Neuropsychopharmacol 19: 1-7, 2016

74. Agnati LF and Fuxe K: Volume transmission as a key feature of information handling in the central nervous system possible new interpretative value of the Turing's B-type machine. Prog Brain Res 125: 3-19, 2000

75. Bao LL, Jiang WQ, Sun FJ, Wang DX, Pan YJ, Song ZX, Wang $\mathrm{CH}$ and Yang $\mathrm{J}$ : The influence of psychological stress on arginine vasopressin concentration in the human plasma and cerebrospinal fluid. Neuropeptides 48: 361-369, 2014.
76. de Winter RF, van Hemert AM, DeRijk RH, Zwinderman KH, Frankhuijzen-Sierevogel AC, Wiegant VM and Goekoop JG: Anxious-retarded depression: Relation with plasma vasopressin and cortisol. Neuropsychopharmacology 28: 140-147, 2003.

77. Speldewinde PC, Cook A, Davies P and Weinstein P: The hidden health burden of environmental degradation: Disease comorbidities and dryland salinity. EcoHealth 8: 82-92, 2011.

78. Chahl LA: TRP's: Links to schizophrenia? Biochim Biophys Acta 1772: 968-977, 2007

79. Kasckow JW, Mulchahey JJ and Geracioti TD Jr: Effects of the vanilloid agonist olvanil and antagonist capsazepine on rat behaviors. Prog Neuropsychopharmacol Biol Psychiatry 28: 291-295, 2004

80. Harrington MG, Salomon RM, Pogoda JM, Oborina E, Okey N, Johnson B, Schmidt D, Fonteh AN and Dalleska NF: Cerebrospinal fluid sodium rhythms. Cerebrospinal Fluid Res 7: 3, 2010.

81. Trotti R, Rondanelli M, Cuzzoni G, Magnani B, Gabanti E and Ferrari E: Circadian organization of serum electrolytes in physiological aging. Funct Neurol 18: 77-81, 2003

82. Sfera A and Osorio C: Water for thought: Is there a role for aquaporin channels in delirium? Front Psychiatry 5: 57, 2014

83. Agnati LF, Zunarelli E, Genedani S and Fuxe K: On the existence of a global molecular network enmeshing the whole central nervous system: Physiological and pathological implications. Curr Protein Pept Sci 7: 3-15, 2006.

84. Wittmann L, Schredl M and Kramer M: Dreaming in posttraumatic stress disorder: A critical review of phenomenology, psychophysiology and treatment. Psychother Psychosom 76: 25-39, 2007.

85. Steenkamp MM, Litz BT, Hoge CW and Marmar CR: Psychotherapy for military-related PTSD: A review of randomized clinical trials. JAMA 314: 489-500, 2015.

86. Polusny MA, Erbes CR, Thuras P, Moran A, Lamberty GJ, Collins RC, Rodman JL and Lim KO: Mindfulness-based stress reduction for posttraumatic stress disorder Among veterans: A randomized clinical trial. JAMA 314: 456-465, 2015.

87. Ganong WF: Review of Medical Physiology. 19th edition. Appleton \& Lange, New York, NY, 1999.

88. Patton HD: Textbook of physiology. Saunders, 21st edition. Saunders, Philadelphia, PA, 1989.

89. Kandel E, Schwartz JH, Jessel TM, Siegelbaum SA and Hudspeth AJ (eds): Principles of neural science. 5th edition. Mac Grow-Hill, New York, NY, 2013. 